\title{
Condición somática de la almeja Polymesoda solida (Veneroidea: Corbiculidae) durante el periodo lluvioso, en el Parque Natural Isla de Salamanca, Caribe colombiano
}

\author{
María Virginia De La Hoz Aristizábal ${ }^{1}$ \\ 1. Instituto Latinoamericano de Ciencias Marinas y del Ambiente (ILCMA) 8101 SW 72 Avenue Suite 420 W, Miami, \\ FL 33143, EEUU. Tel: +1 (786) 797-8105/+1 (786) 223-0844 Fax: +1 (305) 365-4104; mvdelahoz@hotmail.com
}

Recibido 02-VI-2009. Corregido 25-VIII-2009. Aceptado 25-IX-2009.

\begin{abstract}
Somatic condition of the clam Polymesoda solida (Veneroidea: Corbiculidae) in the rainy season of the Salamanca Island Natural Park, Colombian Caribbean. The estuarine clam Polymesoda solida is the main fishery resource in the Salamanca Island Natural Park. I sampled the clam in three interconnected lagoons (Poza Verde, El Torno and Atascosa) during the rainy period (April to November 2004). Sixty individuals were monthly collected by hand on each lagoon; 30 to analyze two condition indexes (relationship between body and shell weight and between soft dry and wet weight), yield (meat percentage) and length-weight relationships. Maturity of female gonad smears was assessed on the remaining individuals. Measures of salinity, temperature, $\mathrm{pH}$, dissolved oxygen, transparency and depth, were also taken at the collection places. Increases of the condition indexes occurred when the Magdalena River showed some decreases in its level, especially at Poza Verde and El Torno. The condition indexes were higher at 27-33mm, (range where maturity size was reached). Clams from Poza Verde, the lagoon nearest to the Magdalena River, had the highest values in condition indexes, yield, fresh weight and maturity frequency. Yield was directly correlated to the condition indexes and sexual maturity. The condition index (body weight/shell weight) showed a highest multiple regression coefficient with salinity, $\mathrm{pH}$ and transparency $\left(\mathrm{R}^{2}=74 \%\right)$, indicating the relevance of the hydrological regime in the regulation of the condition of the organisms. $P$. solida seems to be more adapted to the lowest salinities of the system (0.0-0.4), corresponding to Poza Verde, where the influence of the Magdalena river is more direct and steady. Additionally, differences found between sites in the assessed variables, emphasize the importance of considering spatial heterogeneity of this kind of lagoon estuaries, and should be taken into account for future fisheries management in the rainy period and conservation of P. solida populations. Rev. Biol. Trop. 58 (1): 131-145. Epub 2010 March 01.
\end{abstract}

Key words: Polymesoda solida, bivalves, Salamanca Island, estuaries, hydrological regime, condition index.

La almeja Polymesoda solida Phillippi 1846, es un molusco bivalvo que habita fondos blandos y con cascajo, en aguas estuarinas del continente americano. Ha sido registrada para la parte sur del Caribe y costa norte de Suramérica, desde Belice, hasta el Golfo de Venezuela y Lago de Maracaibo (Cosel 1986, Díaz \& Puyana 1994). El sector occidental de la Isla de Salamanca (SOIS), zona del presente estudio, forma parte de la reserva natural "Vía Parque Isla de Salamanca", donde existen bancos naturales de $P$. solida en varias de sus ciénagas asociadas a manglar (INVEMAR 2004). En la zona de este estudio $P$. solida, es hermafrodita protogínica (Rueda \& Urban 1998), con fecundación externa, desarrollo larval planctónico (García et al. 1994) y se alimenta por filtración de fitoplancton y materia orgánica (De La Hoz 2005).

Actualmente $P$. solida constituye el recurso pesquero más explotado del SOIS, con un $62 \%$ de la captura en peso total de invertebrados y vertebrados comerciales, seguida por la jaiba Callinectes sapidus, con un 18\%; el 20\% 
restante lo ocupan peces de familias como Ariidae Mugilidae, Centropomidae, Cichlidae y Prochilodontidae (INVEMAR 2004); las capturas para el mercado de esta almeja provienen principalmente de las ciénagas Poza Verde, El Torno y Atascosa, las cuales han estado sujetas a explotación desde la década de 1970 (Beleño com. pers.). P. solida se encuentra incluida como "vulnerable" en la lista de especies amenazadas del Caribe colombiano (INVEMAR 2002) y es considerada un indicador de buenas condiciones ambientales en el SOIS, en comparación con el resto de la ecorregión, ya que en la Ciénaga Grande de Santa Marta (CGSM) y el Complejo Pajarales (al oriente del SOIS), afectados por problemas de eutrofización e interrupción de flujos de agua dulce (Botero et al. 1995, Santos-Martínez \& Bateman 1997), se encuentra extinta (Hernández 1983). Con base en estos antecedentes y teniendo en cuenta el monitoreo permanente de la pesquería de la especie, que viene realizándose desde el 2002 en toda la ecorregión (INVEMAR 2008), se puede afirmar que el SOIS constituye un refugio único de esta especie en toda la llanura deltaica del Río Magdalena (RM).

La condición somática de bivalvos, en términos del contenido de biomasa de tejido, ha sido frecuentemente evaluada a través de índices de condición, que generalmente se refieren a la cantidad relativa de carne y son ampliamente utilizados en moluscos para estimar su valor nutritivo, comercial y también ecológico. Tales índices pueden relacionarse con variables como el estado reproductivo, la relación talla-peso, el rendimiento en carne, y permiten conocer cómo responde el organismo a las variaciones del ambiente (Bautista 1989, Acosta et al. 2006). El índice de condición en el género Polymesoda, ha sido estudiado por autores como Ruiz et al. (1998), quienes evaluaron la variación del mismo en relación con el ciclo reproductivo de Polymesoda radiata en Costa Rica. En Colombia, los únicos estudios de $P$. solida, han sido realizados en el Golfo de Urabá (Duque 1993) y en la Isla de Salamanca (Rueda \& Urban 1998, De La Hoz 2005, 2009) y tratan sobre la dinámica poblacional, ecología y pesquería de esta almeja. Sin embargo, no existe algún trabajo en Colombia que evalúe comparativamente el índice de condición de este bivalvo y lo relacione con características biológicas y ambientales. Debido a la importancia económica y ecológica de $P$. solida, el presente trabajo tuvo como objetivo evaluar la condición somática de esta almeja, basándose especialmente en dos índices de condición relacionados con factores biológicos tales como la relación talla-peso, el rendimiento y la frecuencia de madurez sexual y establecer la posible influencia de variables ambientales, en las tres lagunas de mayor importancia para su pesquería en el SOIS.

\section{MATERIALES Y MÉTODOS}

Área de estudio y estaciones de muestreo: El SOIS se encuentra ubicado al este de la desembocadura del RM y al sur del Caribe colombiano $\left(10^{\circ} 43^{\prime}-11^{\circ} 00^{\prime} \mathrm{N}\right.$ y $74^{\circ} 16^{\prime}-74^{\circ} 38^{\prime}$ $\mathrm{W})$, del que lo separa una barra de arena (la Isla de Salamanca). Asimismo se encuentra ratificado desde 1998, en la Convención Relativa a los Humedales de Importancia Internacional RAMSAR (INVEMAR-CORPAMAG 2002). Este sistema lagunar mantiene un intercambio continuo de aguas y sedimentos entre el RM y el Mar Caribe, observándose claramente el aporte del RM en los sedimentos lodosos y arenosos de varias ciénagas. El clima en la zona de estudio, se caracteriza por: una época seca de diciembre a abril, con vientos alisios intensos, predominio de la corriente del Caribe y oleaje fuerte procedente del norte; una época húmeda de mayo a noviembre con vientos alisios débiles, presencia de "vendavales", predominio de la contracorriente del Darién y disminución del oleaje (Erffa 1973).

El presente estudio se llevó a cabo entre abril y noviembre de 2004 en las tres ciénagas más importantes para la pesquería de $P$. solida en el SOIS (Poza Verde, El Torno y Atascosa). Poza Verde, al occidente, mide aproximadamente $4.5 \mathrm{~km}^{2}$ y recibe la influencia más directa del RM a través del caño Almendros. El Torno se comunica directamente con Poza Verde, 
hacia el noreste, tiene una extensión de $7 \mathrm{~km}^{2}$; le siguen otras ciénagas comparativamente pequeñas e interconectadas donde no se lleva a cabo explotación de P. solida (El Loro, Las Piedras, Tronconera, Palmichal). Atascosa, conectada con Palmichal hacia el oriente, tiene una extensión aproximada de $3.6 \mathrm{~km}^{2}$, está separada del Mar Caribe por la Isla de Salamanca y es la que recibe mayor influencia marina. Mas detalles sobre la geomorfología de la región, son descritos por Erffa (1973) y Bernal (1996). En cada una de las tres lagunas de este estudio, hay una "zona de pesca" o banco natural ubicado lejos de la orilla, con menos de tres metros de profundidad y que contiene la mayor densidad de almejas. Dichos bancos naturales han sido escogidos por los pescadores artesanales para la extracción del recurso (De La Hoz 2005).

Toma de muestras y análisis de la información: Se recolectaron 60 individuos de $P$. solida manualmente al azar, en forma mensual (abril a noviembre de 2004) en cada estación (ciénaga), utilizando el muestreo por conglomerados (Ospina 2001), en la zona de pesca de cada una de las tres ciénagas; de este modo se realizaron tres muestreos por mes, resultando en total 24 muestreos y 1440 ejemplares en los ocho meses de estudio, que abarcaron el periodo lluvioso. Como medio de transporte se utilizó una lancha con motor fuera de borda, entrando desde el RM por el caño Almendros hacia Poza Verde y de ahí se hizo el traslado por los demás caños que interconectan las ciénagas restantes. Los bajos niveles de agua en los tres primeros meses del año, impidieron el acceso a los sitios de muestreo. De cada muestra de 60 individuos por estación, una submuestra de 30 se transportó refrigerada y fue utilizada para obtener los pesos de los organismos y la otra submuestra de 30 fue transportada con los organismos vivos en recipientes con agua de las mismas ciénagas para observar la madurez sexual. En los lugares de muestreo se midieron las variables ambientales temperatura $\left({ }^{\circ} \mathrm{C}\right)$ y salinidad (UPS) con un equipo Conduct YSI MOD: EC300, pH con un pH-metro WTW MOD: PH320 y oxígeno disuelto $(\mathrm{mg} / \mathrm{L})$ con un oxímetro WTW MOD: OXI320; la transparencia $(\mathrm{cm})$ y la profundidad de la columna de agua $(\mathrm{cm})$, se tomaron con un disco Secchi; estas variables fueron medidas en el agua de fondo, por ser la que está en contacto directo con las almejas. También se obtuvo información del nivel del RM para cada mes (CORPAMAG-UNINORTE 2005).

Los pesos húmedo y seco del cuerpo y de la concha se determinaron con una balanza analítica de $0.0001 \mathrm{~g}$ de precisión; los pesos secos de la concha limpia y del cuerpo, se obtuvieron por secado en la estufa a $60^{\circ} \mathrm{C}$ hasta peso constante (Holme \& Mc Intyre 1971). A partir de estos pesos se calculó el peso total, el rendimiento y dos índices de condición (IC e IC2):

IC $(\%)=($ peso seco cuerpo/peso seco concha)*1000 (Bautista 1989, García 1997)

IC2 $(\%)=($ peso seco cuerpo/peso húmedo cuerpo)*100 (Bautista 1989)

Peso total $(\mathrm{g})=$ peso húmedo cuerpo + peso concha

Rendimiento $(\%$ carne $)=$ (peso húmedo cuerpo/ peso total)*100 (Cabrera et al. 2001, Silva \& Bonilla 2001).

En la submuestra de organismos vivos se observaron al microscopio los óvulos maduros en los frotis en fresco del material gonadal. Se consideró como indicativo de madurez una llenura de gónada de $75 \%$ a $100 \%$ de óvulos en el campo visual. Los individuos maduros se diferenciaron de los restantes en que aquellos presentaban $50 \%$ o más folículos postovulatorios (vacíos) o bien, carecían de células sexuales. Al representar gráficamente el porcentaje de madurez por tallas, se ubicó la talla de madurez del 50\% y del $75 \%$ de la población. King (1995) recomienda este método en fresco para evaluar en forma rápida la condición reproductiva en recursos pesqueros, en estas células de mayor tamaño, cuando no se dispone de técnicas más sofisticadas. La longitud valvar anteroposterior (L) de todos los individuos, se 
midió con un calibrador Vernier de $0.05 \mathrm{~mm}$ de precisión.

Para determinar diferencias en los índices de condición entre muestreos $(\mathrm{p}<0.05)$, se realizaron ANOVAS de Kruskal-Wallis; y para determinar diferencias en los índices de condición entre las ciénagas se realizaron ANOVAS de Friedman por observarse que las variables no cumplían con los supuestos de normalidad en todos los casos según pruebas de Shapiro-Wilks, Kolmogorov-Smirnov y chi ${ }^{2}$ efectuadas a priori $(\mathrm{p}<0.05)$ (Sokal \& Rohlf, 1981). Mediante correlaciones de Spearman se determinó si existía una asociación conjunta significativa $(\mathrm{p}<0.05)$ de los índices de condición con el porcentaje de individuos maduros, el rendimiento y las variables ambientales. Se calculó la relación talla-peso en cada ciénaga, utilizando el peso húmedo y el peso total, mediante regresión potencial $\left(\mathrm{W}=\mathrm{L}^{\mathrm{b}}\right)$ y se realizó la prueba t para determinar el crecimiento isométrico $(b=3)$ o alométrico $(b \neq 3)$, con base en la ecuación de Pauly (Zar 1999).

A través de una regresión múltiple, se establecieron las variables independientes que explicaron mejor la condición física de la almeja, utilizando como variable dependiente el índice de condición (IC1 o IC2) que hubiera mostrado mejores correlaciones con las variables ambientales. La significancia de la regresión se estableció por medio de un análisis de varianza con una prueba $\mathrm{F},(\mathrm{p}<0.05)$ y el grado de ajuste se obtuvo a través del coeficiente de regresión $\mathrm{R}^{2}$ (Sokal \& Rohlf 1981). Para todos los análisis estadísticos se utilizó el programa STATISTICA v. 5.5. ®

Los especímenes de referencia de $P$. solida se encuentran en la colección del museo de historia natural marina del Instituto de Investigaciones Marinas y Costeras INVEMAR, con número de catálogo MOL-1235-7129, con información disponible en línea (SIBM 2009).

\section{RESULTADOS}

El sistema estuarino donde habita P. solida, se caracterizó por la amplia variabilidad especialmente entre las ciénagas, en la salinidad y en la transparencia del agua. En Poza Verde (ciénaga más cercana al RM) se observaron los menores valores de estas dos variables $(0.16 \pm 0.12$ UPS y $18.38 \pm 4.44 \mathrm{~cm}$, respectivamente); El Torno presentó la mayor transparencia $(67.50 \pm 14.64 \mathrm{~cm})$ y una salinidad de 0.44 \pm 0.11 UPS; Atascosa (ciénaga más cercana al mar) presentó la mayor salinidad $(7.13 \pm 5.37$ UPS $)$ y una transparencia de $37.50 \pm 10.69 \mathrm{~cm}$ (Fig. 1). Las siguientes variables mostraron una variabilidad relativamente baja, en Poza Verde, El Torno y Atascosa respectivamente: temperatura $\left(29.38 \pm 0.3^{\circ} \mathrm{C}, 29.48 \pm 0.9^{\circ} \mathrm{C}\right.$ y $\left.29.95 \pm 0.5^{\circ} \mathrm{C}\right)$, pH $(7.57 \pm 0.21,7.84 \pm 0.19$ y $8.29 \pm 0.28)$, oxígeno disuelto $(7.05 \pm 0.58 \mathrm{mg} / 1,7.02 \pm 0.57 \mathrm{mg} / 1$ y $7.75 \pm 0.99 \mathrm{mg} / \mathrm{l})$ y profundidad de la columna de agua $(123.75 \pm 26.3 \mathrm{~cm}, 113.13 \pm 60.23 \mathrm{~cm}$ y $121.25 \pm 22.95 \mathrm{~cm})$.

La almeja presentó los mayores pesos húmedos y secos en promedio en Poza Verde, pero los mayores pesos totales los alcanzó en Atascosa, donde también presentó el mayor peso de la concha y la mayor talla promedio. En El Torno se presentaron los menores pesos del cuerpo, de las conchas y la menor talla promedio. El mayor rendimiento se presentó en Poza Verde y el menor en Atascosa (Cuadro 1). Los intervalos de tallas predominantes (L)

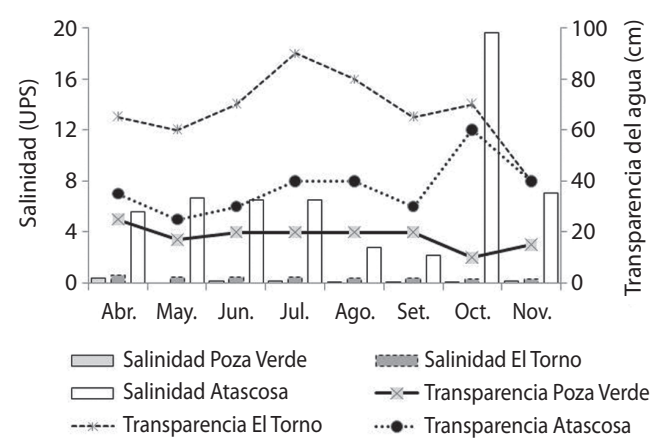

Fig. 1. Salinidad y transparencia del agua (disco Secchi) en las tres ciénagas de $P$. solida en el sector occidental de la Isla de salamanca, entre abril y noviembre 2004.

Fig.1. Water salinity and transparency (Secchi disk) at the three lagoons of $P$. solida in the Salamanca Island occidental sector, between April and November 2004. 
CUADRO 1

Pesos (g), rendimiento (\% de carne) y talla promedio $(\mathrm{mm})$ de P. solida en las ciénagas Poza Verde, El Torno y Atascosa

TABLE 1

Weights (g) yield (\% meat) and mean size ( $\mathrm{mm}$ ) of P. solida at the Poza Verde, El Torno and Atascosa lagoons

$\begin{array}{llcccccccccc}\text { Variable } & \text { Ciénaga } & \text { Abr } & \text { May } & \text { Jun } & \text { Jul } & \text { Ago } & \text { Sep } & \text { Oct } & \text { Nov } & \text { Promedio } \\ \text { Peso } & \text { Poza Verde } & 1.37 & 2.44 & 1.09 & 1.97 & 1.92 & 1.87 & 1.48 & 2.10 & 1.78 \\ \text { húmedo cuerpo } & \text { El Torno } & 1.44 & 0.81 & 0.75 & 0.75 & 1.50 & 1.67 & 1.23 & 2.10 & 1.28 \\ & \text { Atascosa } & 0.96 & 1.19 & 1.13 & 1.41 & 1.63 & 1.73 & 1.60 & 1.77 & 1.43 \\ \text { Peso concha } & \text { Poza Verde } & 5.41 & 8.36 & 4.14 & 6.67 & 5.84 & 7.04 & 5.56 & 7.79 & 6.35 \\ \text { (seca) } & \text { El Torno } & 5.74 & 4.53 & 3.91 & 4.43 & 5.96 & 6.63 & 5.31 & 8.52 & 5.63 \\ & \text { Atascosa } & 6.71 & 7.68 & 7.35 & 8.17 & 8.79 & 7.67 & 8.78 & 8.02 & 7.90 \\ \text { Peso seco cuerpo } & \text { El Torno } & 0.21 & 0.09 & 0.10 & 0.08 & 0.19 & 0.27 & 0.21 & 0.31 & 0.18 \\ & \text { Atascosa } & 0.13 & 0.13 & 0.14 & 0.16 & 0.23 & 0.25 & * & 0.29 & 0.17 \\ \text { Peso total } & \text { Poza Verde } & 7.60 & 11.65 & 6.26 & 9.27 & 9.11 & 10.42 & 8.39 & 10.76 & 9.18 \\ & \text { El Torno } & 7.68 & 5.32 & 4.92 & 5.41 & 8.40 & 8.61 & 7.36 & 11.35 & 7.38 \\ \text { Talla } & \text { Atascosa } & 7.97 & 9.20 & 8.83 & 9.98 & 11.41 & 10.19 & 10.38 & 10.47 & 9.80 \\ \text { promedio } & \text { Poza Verde } & 27.63 & 30.12 & 26.94 & 31.16 & 30.06 & 30.89 & 28.61 & 32.18 & 29.70 \\ & \text { El Torno } & 29.42 & 26.92 & 25.08 & 26.09 & 29.20 & 29.57 & 27.65 & 33.01 & 28.37 \\ \text { Rendimiento } & \text { Atascosa } & 31.45 & 31.69 & 31.52 & 32.28 & 32.25 & 31.45 & 31.98 & 32.26 & 31.86 \\ & \text { Poza Verde } & 18.05 & 20.98 & 17.35 & 21.28 & 21.13 & 17.93 & 17.67 & 19.52 & 19.24 \\ & \text { El Torno } & 18.75 & 15.15 & 15.29 & 13.81 & 17.87 & 19.36 & 16.73 & 18.53 & 16.94\end{array}$

* Ejemplares insuficientes por muerte masiva.

fueron 25-30mm en Poza Verde y El Torno y 30-35mm en Atascosa.

Los mayores índices de condición (IC e IC2) en promedio también correspondieron a Poza Verde ( $\mathrm{IC}=48.4 \%, \mathrm{IC} 2=17.41 \%$ ), seguidos por El Torno ( $\mathrm{IC}=30.69 \%$, IC2 $=14.41 \%$ ) y por Atascosa (IC $=24.66 \%, I_{C}=13.47 \%$ ) (Fig. 2A-C). Ambos fueron significativamente diferentes entre las ciénagas (IC: Friedman=13.0, $\mathrm{p}<0.05$, IC2: Friedman $=9.3, \mathrm{p}<0.05)$ y entre los muestreos (IC: Kruskal-Wallis=81 (Poza Verde), 159 (El Torno), 158.7 (Atascosa). IC2: Kruskal-Wallis=77.1 (Poza Verde), 113.1 (El Torno), 110 (Atascosa), p<0.05). La Fig. 3, ilustra a modo comparativo la variación de la profundidad de la columna del agua en los puntos de muestreo y del nivel del RM (medido en cotas sobre el margen derecho del río), siendo ambos menores al comienzo del estudio (marzo a mayo) y mayores en la segunda mitad del año; no obstante, no se encontró correlación significativa entre estas dos variables (Spearman $\mathrm{p}>0.05)$. Los índices de condición en Poza Verde y El Torno, tendieron a un aumento especialmente en abril, agosto y septiembre coincidiendo con descensos en el nivel de este río, y presentaron los valores más bajos en junio, julio, octubre y noviembre, coincidiendo con ascensos en el nivel (Fig. 2 A-B). En Atascosa, se mantuvieron bajos en la primera mitad del estudio y aumentaron entre agosto y noviembre (Fig. 2C), coincidiendo este último con el mayor nivel del RM. Sin embargo, estos índices no mostraron correlación con dicho nivel (Spearman, $\mathrm{p}>0.05$ ). Se resalta que la muerte masiva de almejas sucedida en octubre en Atascosa, que coincidió con un aumento brusco de salinidad (Fig. 1), impidió recolectar individuos suficientes para el análisis de los índices de condición de esta ciénaga, en ese 

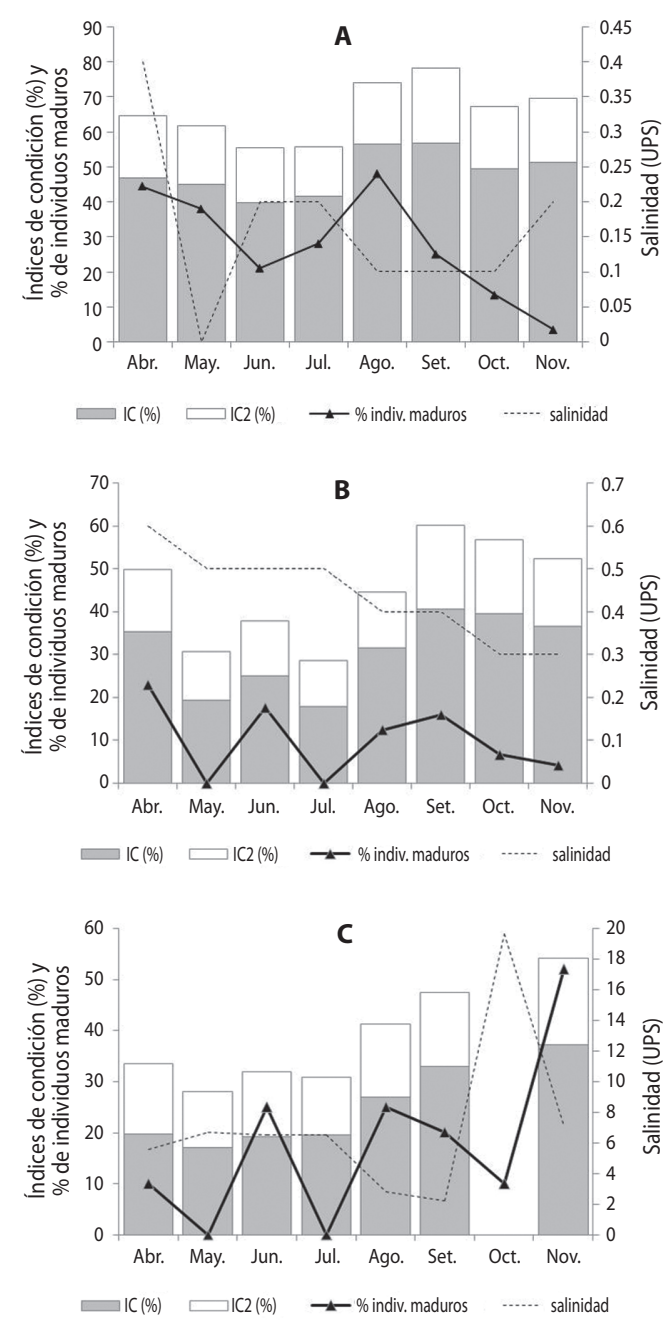

Fig. 2. Índices de condición IC e IC2 (\%) y frecuencia de madurez sexual (\%) (con base en la gónada femenina) de $P$. solida en las tres ciénagas del sector occidental de la Isla de Salamanca, abril a noviembre de 2004. (A.) Poza Verde, (B.) El Torno, (C.) Atascosa. Se compara con la salinidad del agua en cada ciénaga.

Fig. 2. Condition indexes IC and IC2, and sexual maturity frequency (\%) (based on the female gonad) of $P$. solida, at the three lagoons in the Salamanca Island occidental sector, April to November 2004. (A.) Poza Verde, (B.) El Torno, (C.) Atascosa. It is compared to the water salinity at each lagoon.

mes. El IC y el IC2 se correlacionaron inversamente con la salinidad, el $\mathrm{pH}$ y la transparencia del agua (Spearman $\mathrm{p}<0.05$ ), con coeficientes

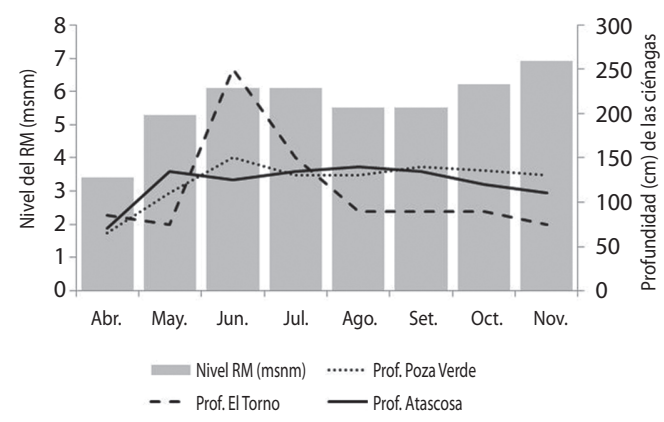

Fig. 3. Niveles del Río Magdalena entre abril y noviembre de 2004 (CORPAMAG- UNINORTE 2005). Se indica la profundidad del agua en las tres lagunas.

Fig. 3. Magdalena river levels from April to November 2004 (CORPAMAG - UNINORTE 2005). Water depth is indicated at each lagoon.

relativamente altos $(\mathrm{R}>0.60)$; mientras con los demás factores ambientales no hubo correlación significativa. Los pesos del cuerpo, de la concha y el rendimiento (Cuadro 1), exhibieron un patrón de variación similar a los dos índices de condición; no obstante, estos índices estuvieron correlacionados significativamente solamente con el peso húmedo del cuerpo y con el rendimiento (Spearman, $R>0.60, \mathrm{p}<0.05$ ).

El porcentaje de individuos maduros fue significativamente diferente entre las ciénagas (Friedman $=6.7, \mathrm{p}<0.05$ ), con un promedio de $27.6 \%$ en Poza Verde, $10 \%$ en El Torno y $17.8 \%$ en Atascosa. En el resto de los ejemplares, una parte importante de la gónada ya estaba ocupada por folículos postovulatorios, o éstos se encontraban en reabsorción, indicando etapas posteriores o de desove, que no fue posible precisar con la técnica empleada. En Poza verde y El Torno, esta frecuencia tendió a incrementarse en época de menores niveles del RM (abril, mayo y agosto), y a presentar los valores más bajos en octubre y noviembre (Fig. 2 A-B), cuando este río alcanza los mayores niveles (Fig. 3). Atascosa difiere de las ciénagas anteriores en que los porcentajes de individuos maduros fueron mayores cuando los niveles del RM aumentaron (Fig. 2C, Fig. 3). El patrón de variación del porcentaje 
de individuos maduros fue similar al de los índices de condición, correlacionándose en forma directa (Spearman, $\mathrm{p}<0.05$ ), aunque con coeficientes no muy altos $(\mathrm{R}<0.60)$. Los individuos maduros estuvieron presentes en todos los muestreos y en todas las ciénagas. Asimismo, los individuos con mayores llenuras de gónada y mayores índices de condición, se caracterizaron por poseer una masa visceral turgente y de aspecto cremoso o blanquecino con un frotis gonadal de consistencia granulosa, mientras que en los individuos con menores índices de condición y menor llenura de gónada, la masa visceral era flácida, de aspecto traslúcido y el frotis era de consistencia lisa.

La madurez sexual de $P$. solida se evaluó solamente en la gónada femenina, ya que las observaciones de espermatozoides fueron aisladas y poco claras, presentándose sólo en el 9\% de los individuos. Estos presentaban cantidad despreciable o ausencia de óvulos, o también unos cuantos folículos vacíos e inclusiones lipídicas, indicando reabsorción de la gónada femenina después del desove, corroborando el hermafroditismo de esta especie. La gónada femenina activa se observó en $66 \%$ de los organismos, mientras el $25 \%$ restante, por su ausencia de contenido gonadal, se asoció a etapas posteriores al desove.

Por otro lado, los índices de condición en las tres ciénagas van aumentando a medida que los individuos son más grandes, hasta las tallas más frecuentes en el estudio (25-35mm), alcanzando los mayores valores en el intervalo de 27-33mm, para luego comenzar a disminuir en tallas mayores de $33 \mathrm{~mm}$ (Fig. 4a, b y c). Para una mejor visualización, en las tallas más comunes $(25-35 \mathrm{~mm})$ los índices de condición se representaron a intervalos de $2 \mathrm{~mm}$ y debido al bajo número de ejemplares, a intervalos más amplios para las tallas menores de $25 \mathrm{~mm}$ y mayores de $35 \mathrm{~mm}$. Adicionalmente, se encontró que las tallas de madurez del 50\% y del 75\% de los ejemplares $\left(\mathrm{L}_{50} \mathrm{y} \mathrm{L}_{75}\right)$, estuvieron ubicadas dentro del intervalo de tallas con mayores índices de condición (Fig. 5). A pesar de las tendencias observadas, los índices de condición no se correlacionaron significativamente con la talla de los individuos (Spearman, $\mathrm{p}>0.05$ ).

La talla de $P$. solida no se correlacionó con el peso seco del cuerpo, pero sí directamente con los pesos húmedo y total (Spearman, $\mathrm{p}<0.05, \mathrm{R}>0.60$ ). La relación talla-peso total, señaló crecimiento isométrico en los individuos de Poza Verde $\left(\mathrm{W}=0.000 \mathrm{~L}^{2.993}\right.$, $\left.\mathrm{R}^{2}=0.91\right)$ y alométrico negativo en los de El Torno $\left(\mathrm{W}=0.0006 \mathrm{~L}^{2.8255}, \mathrm{R}^{2}=0.90\right)$ y Atascosa $\left(\mathrm{W}=0.0021 \mathrm{~L}^{2.4392}, \mathrm{R}^{2}=0.80\right)$. Asimismo, la relación talla-peso húmedo del cuerpo, señaló crecimiento alométrico positivo para Poza Verde $\left(\mathrm{W}=1 \mathrm{E}-05 \mathrm{~L}^{3.4146}, \mathrm{R}^{2}=0.83\right)$ y para $\mathrm{El}$ Torno $\left(\mathrm{W}=4 \mathrm{E}-06 \mathrm{~L}^{3.7311}, \mathrm{R}^{2}=0.77\right)$ y alométrico negativo para Atascosa $\left(\mathrm{W}=0.0002 \mathrm{~L}^{2.5377}\right.$, $\mathrm{R}^{2}=0.53$ ). En general, todas las regresiones fueron significativas $(\mathrm{p}<0.05)$.

Debido a que presentó las mayores correlaciones con los factores fisicoquímicos, se escogió al IC como variable dependiente para evaluar mediante regresión múltiple, la influencia de dichos factores sobre la condición somática de la almeja en las tres ciénagas en conjunto. Las salinidad, $\mathrm{pH}$ y transparencia del agua en conjunto, explicaron el comportamiento del IC, con el mejor ajuste $\left(\mathrm{R}^{2}=74 \%, \mathrm{p}<0.05\right)$, expresado por la ecuación: $\mathrm{IC}=156.73-1.88^{*}$ salinidad-13.70* pH-0.257375* transparencia. La temperatura, oxígeno disuelto y profundidad de la columna de agua, presentaron la menor influencia sobre el valor del IC ( $p>0.05)$.

\section{DISCUSIÓN}

La amplia variabilidad de los factores fisicoquímicos evaluados (Fig. 1), corrobora el alto dinamismo del estuario donde habita $P$. solida (INVEMAR-CORPAMAG 2002). Las salinidades progresivamente más altas a medida que las ciénagas del SOIS se encuentran a mayor distancia del RM y más cercanas al mar, muestra el patrón de influencia de estas dos fuentes de agua, típica en sistemas lagunares estuarinos, con valores particulares para cada cuerpo de agua (INVEMAR-CORPAMAG 2002, Gocke et al. 2003). La alta variabilidad temporal y espacial de la salinidad, lleva a 
A

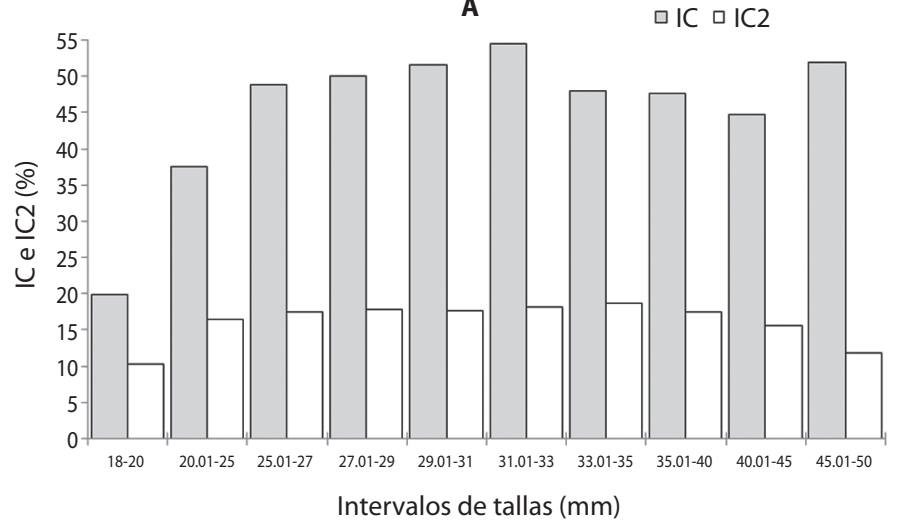

B $\quad \square I C \square I C 2$

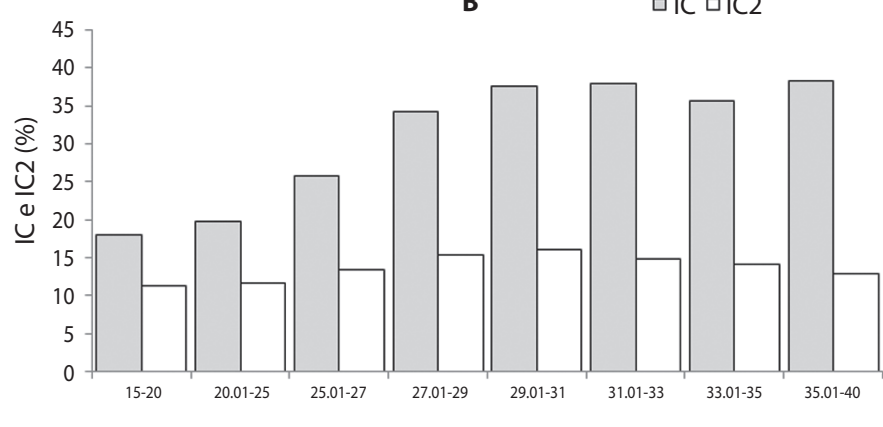

Intervalos de tallas $(\mathrm{mm})$

C

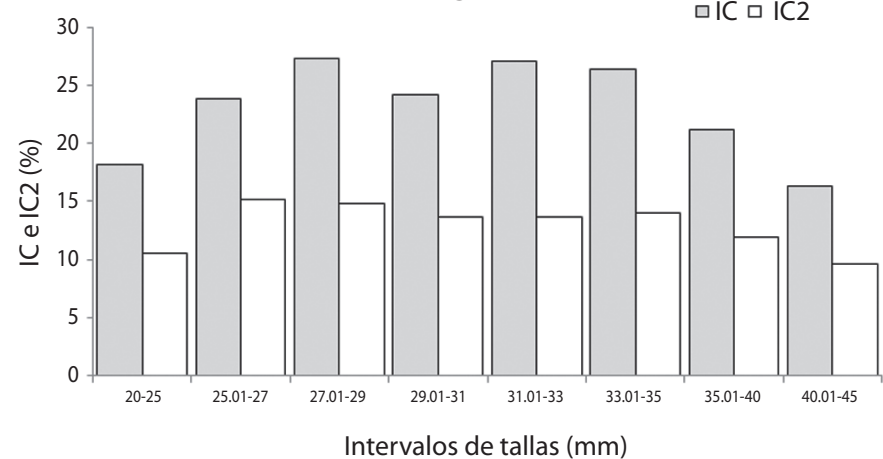

Fig. 4. Distribución de los índices de condición IC e IC2 por intervalo de tallas de P. solida en el sector occidental de la Isla de Salamanca, abril a noviembre 2004. (A.) Poza Verde, (B.) El Torno, (C.) Atascosa.

Fig. 4. Distribution of the condition indexes IC and IC2 by size interval of $P$. solida in the Salamanca Island occidental sector, April to November 2004. (A.) Poza Verde, (B.) El Torno, (C.) Atascosa. 


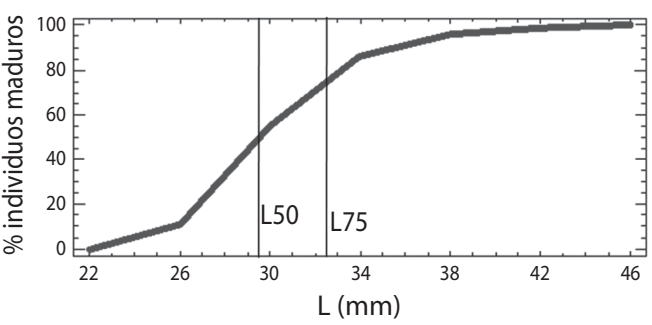

Fig. 5. Frecuencia acumulada de tallas de P. solida, indicando la talla de madurez del 50\% (L50) y del 75\% de los ejemplares (L75), en el sector occidental de la Isla de Salamanca, entre abril y noviembre de 2004.

Fig. 5. Accumulative size frequency of $P$. solida, showing maturity size for the 50\% (L50) and 75\% (L75) of the specimens, at the Salamanca Island western sector, between April and November 2004.

clasificar a las lagunas Poza Verde y El Torno en el ámbito de limnética a oligohalina y a Atascosa en el ámbito de mesohalina de acuerdo con Bulger et al. (1993). Por su parte, el pH se mantuvo dentro del intervalo de valores para estuarios no contaminados (5-9), mientras que los niveles de oxígeno disuelto fueron superiores a la concentración mínima tolerada por los seres vivos (2ml/l) (Hernández 1988, INVEMAR-CORPAMAG 2002), indicando que las condiciones ambientales en las que habita $P$. solida son relativamente buenas. Dado que la salinidad refleja el grado en el que el agua de mar que entra en el estuario ha sido diluida por la llegada de agua dulce, se puede decir que el SOIS posee la característica habitual de los estuarios de tipo lagunar, de recibir un alto aporte de agua dulce (Olsen et al. 2009), lo que resalta la importancia del RM como subsidio vital de energía para el mantenimiento de comunidades biológicas como la de esta almeja. Los niveles de este río, se han caracterizado a través de los años por sus aumentos y disminuciones en respuesta a la variación de las precipitaciones entre las épocas climáticas seca y húmeda (Fig. 3) (IDEAM 2003).

Las correlaciones inversas de los dos índices de condición de $P$. solida con la salinidad y la transparencia del agua, pueden explicarse porque en lugares con menor transparencia, como Poza Verde, hay mayor concentración de material suspendido y por lo tanto mayor disponibilidad de alimento (Hernández 1983); registros de sólidos suspendidos durante el tiempo de estudio, muestran valores en promedio mayores para Poza Verde (144.9mg/l), seguidos de Atascosa $(70.7 \mathrm{mg} / \mathrm{l})$ y El Torno $(23.9 \mathrm{mg} / \mathrm{l})$. Por otro lado, la concentración de clorofila-a, aunque muestra mayor promedio en Atascosa $(30.8 \mu \mathrm{g} / \mathrm{l})$ que en Poza Verde $(21 \mu \mathrm{g} / \mathrm{l})$, en El torno se da el menor promedio $(8.2 \mu \mathrm{g} / \mathrm{l})$, coincidiendo con la mayor transparencia del agua (Henry com. pers.). Poza Verde se caracterizó por la menor salinidad y los mayores índices de condición, contrario a Atascosa, que presentó una mayor salinidad. Esto sugiere una mejor condición somática para $P$. solida en Poza Verde, dada la mayor proporción de carne respecto a la concha en el IC, a la cantidad de agua en los tejidos en el IC2 y al peso total (rendimiento) (Cuadro 1); Rueda \& Urban (1998) señalan a esta ciénaga como la de mayor producción de la especie en la zona y en este estudio se observó como la más utilizada para la extracción de $P$. solida, con presencia del mayor número de pescadores por día (Beleño com. pers.), lo que puede relacionarse con las cualidades biológicas aquí evaluadas.

Se ha mencionado la utilidad de los índices de condición y del peso corporal, como indicativos de desove cuando disminuyen y de madurez cuando aumentan (Bautista 1989, Solano et al. 1997, Rueda \& Urban 1998). Además, Duque (1993) menciona que la gónada en desarrollo avanzado de $P$. solida constituye un porcentaje alto de su peso total. De ahí la correlación directa observada entre los índices de condición y la frecuencia de individuos maduros en la almeja en el SOIS, donde el bajo porcentaje de ejemplares maduros en El Torno y Atascosa, coincide con índices de condición más bajos que en Poza Verde, lo que significa un menor rendimiento (Cuadro 1).

La asociación conjunta directa y significativa de la frecuencia de madurez con los índices de condición, ha sido informada en otros lugares del trópico, tal como el IC2 de $P$. radiata en Costa Rica (Ruiz et al. 1998) y de 
Arca zebra en Venezuela (Prieto et al. 2001). Es por esto que los máximos índices de condición y por tanto los mayores rendimientos, tienden a presentarse en tallas en que los organismos generan más tejido reproductivo, y que por lo general se establece en tallas que no son las más grandes, como lo mencionan Silva \& Bonilla (2001) para el género Anadara y Solano et al. (1997) para Pinctada mazatlanica. La fecundidad disminuye con la edad de las almejas. Por eso los mayores índices de condición de $P$. solida en el intervalo de $25-35 \mathrm{~mm}$, concuerdan con el mayor porcentaje de almejas maduras en ese mismo intervalo (Fig. 5). De ahí que las diferencias significativas entre meses, en los índices de condición, pueden asociarse a la a la forma como la frecuencia de individuos maduros varía mensualmente en $P$. solida. Dada la presencia de individuos maduros de $P$. solida en todos los meses de muestreo, existe la posibilidad de actividad reproductiva continua, como se ha señalado típicamente en recursos pesqueros tropicales (Sparre \& Venema 1995, Báez et al. 2005), ya que Rueda \& Urban (1998) observaron individuos maduros de esta especie en todos los meses durante un año completo, aunque sólo en Poza Verde. Por lo tanto, la reproducción continua de la especie deberá corroborarse en las tres ciénagas abarcando todo un año, para llegar a una conclusión definitiva. Debido a que $P$. solida en el SOIS alcanza su madurez sexual masculina y femenina a los $16 \mathrm{~mm}$ y a los $21 \mathrm{~mm}$ respectivamente (Rueda \& Urban 1998) y dada la observación casi exclusiva de óvulos, en individuos que en su mayoría midieron más de $20 \mathrm{~mm}$, existe la posibilidad de un hermafroditismo consecutivo en esta almeja, que no fue posible constatar durante los ocho meses de estudio en el SOIS, ya que el cambio de sexo puede tardar hasta un año completo (Severeyn com. pers.). Además, hay que considerar que la técnica utilizada sólo permite estimar de manera muy general la variación de la madurez de la gónada femenina y no permite conocer en detalle la gónada masculina, como para asociar la variación de la madurez de ésta con los índices de condición, para lo que sería indispensable el uso de técnicas histológicas (King 1995, Ruiz et al. 1998).

La presencia de mayores índices de condición en tallas intermedias, también puede ser debida a que en los bivalvos, a partir de cierto tamaño la producción de carne cesa y únicamente continúa creciendo la concha y aumentando su grosor por acumulación de carbonato de calcio, lo que aumenta acentuadamente el peso de la concha, como lo mencionado por Duque (1993) para $P$. solida; y además se refleja en la disminución del rendimiento para Anadara tuberculosa y A. similis, como lo reportado por Silva \& Bonilla (2001). P. solida en el SOIS, sigue esta misma tendencia, con índices de condición menores en tallas menores, de $18 \mathrm{~mm}$ a $25 \mathrm{~mm}$, y mayores, de $33 \mathrm{~mm}$ a $45-50 \mathrm{~mm}$ (Fig. 4), que es por lo general el intervalo más grande que puede encontrarse para esta especie en el SOIS (De La Hoz 2009).

Comparando magnitudes de índices de condición entre diferentes especies de bivalvos, Ruiz et al. (1998) estiman el IC2 para $P$. radia$t a$, entre $7.75 \%$ y $12.03 \%$ (9.89\% en promedio), mientras que en $P$. solida en el SOIS dicho índice varió de $10 \%$ a $21 \%$ en promedio mensual, lo que muestra el potencial comparativamente alto de la especie como recurso alimenticio. En otras especies, como A. zebra en Venezuela se ha encontrado mayores valores para este índice (Prieto et al. 2001), variando entre $15 \%$ y $28 \%$. El valor económico y alimenticio de $P$. solida en el SOIS serían favorecidos cuanto mayores sean los índices de condición (Bautista 1989), que en el caso de este estudio, tales valores hacen suponer mayores ventajas para las almejas de Poza Verde en cuanto a la calidad del producto, respecto a las de las ciénagas El Torno y Atascosa.

Asimismo, el rendimiento de $P$. solida en el SOIS, es alto en comparación con otras especies. Cabrera et al. (2001) registran un promedio de $10.32 \%$ para Saccostrea palmula en Costa Rica y Squires et al. (1978) registran un promedio de $15 \%$ para $A$. tuberculosa en Colombia, que supera únicamente al promedio encontrado en Atascosa en este estudio. Por otro lado, Silva \& Bonilla (2001) reportan un 
rendimiento alto en $A$. tuberculosa $(17.2 \%)$ y en $A$. similis (21.2\%), este último superando a los promedios de las almejas de las tres lagunas del SOIS.

El rendimiento en bivalvos se refiere a la capacidad para producir la máxima cantidad de carne, al igual que los índices de condición IC e IC2, sirven para indicar la calidad del producto y reflejan la actividad fisiológica y va acorde a un patrón de variación similar del IC y del IC2 en este estudio. Adicionalmente, la mayor turgencia y aspecto cremoso en la masa visceral de la almeja, puede asociarse a una mejor calidad de la carne, coincidiendo con los índices de condición y rendimiento más alto, como se observó en Poza Verde, al contrario de individuos con masas viscerales flácidas y traslúcidas. Estas características ya han sido anteriormente consideradas para evaluar la calidad de la carne en otros bivalvos comestibles, como los mejillones (Acosta et al. 2006).

La variabilidad temporal en la condición somática de la almeja, puede asociarse a los cambios estacionales en zonas tropicales con sus variaciones de salinidad (Báez et al. 2005, García et al. 1994) y donde el RM podría estar cumpliendo una función importante. Si bien los cambios estacionales no se vieron en el año completo, el nivel del RM sí presentó cambios asociados a las llamadas época lluviosa menor y época lluviosa mayor, característico del régimen climático del Caribe colombiano (Erffa 1973); por eso entre abril y julio el nivel del RM fue menor en promedio que entre agosto y noviembre (Fig. 3). A su vez, la variabilidad entre sitios en las características biológicas, puede relacionarse con el gradiente espacial de influencia de dicho río, que se refleja en marcadas diferencias espaciales de salinidad (INVEMAR-CORPAMAG 2002). La importancia de la salinidad en sistemas estuarinos, es bien conocida por regir aspectos físicos y reproductivos de los organismos (Bulger et al. 1993, INVEMAR 2004) y dado que la regresión múltiple mostró que la salinidad, $\mathrm{pH}$ y transparencia del agua influían en forma significativa en la condición somática con base en el IC, es de suponer algún efecto importante del régimen hidrológico en la regulación de dicha condición de la almeja, a pesar de la ausencia de correlación del nivel del RM con las demás variables evaluadas. Esto podría explicarse por dos razones: Primero, el dragado eficiente de caños efectuado en el 2002 debido a la alta sedimentación de la zona, pudo haber permitido la permanencia de agua dulce por más tiempo en el sistema y en segundo lugar, la presencia en el 2004 de caudales del RM superiores al caudal medio multianual en respuesta al aumento de precipitaciones en el interior del país (CORPAMAG-UNINORTE 2005). Tales eventos permitieron mantener las salinidades muy cercanas a cero en el 2004, enmascarando así una posible correlación del régimen hidrológico con la salinidad y con otras variables bióticas y abióticas.

Por otro lado, la importancia de la transparencia sobre el comportamiento del IC, puede atribuirse al contenido de seston total en el agua por los aportes fluviales, contribuyendo a la producción primaria del sistema (Gocke et al. 2003, Hernández 1983, 1988) y siendo altamente aprovechable por los animales filtradores en los estuarios (Schweers et al. 2006). Durante el periodo lluvioso, los índices de condición aumentan a una menor transparencia del agua, como lo indicó la correlación inversa, con un mayor beneficio para la condición somática de la especie en Poza Verde, resaltando de nuevo la posible importancia del RM. En general la influencia del régimen hidrológico de los estuarios es determinante de las condiciones ambientales que repercuten en las actividades biológicas de los propios organismos (INVEMAR-CORPAMAG 2002; Gocke et al. 2003, Olsen et al. 2009).

Por otro lado, durante el periodo estudiado el oxígeno y la temperatura no sugieren influencia significativa sobre la condición de $P$. solida, presentando poca influencia en la regresión múltiple, debido posiblemente a que el oxígeno es tolerado por este género en intervalos muy amplios de concentración (Duque 1993) y que en el SOIS siempre estuvo alto. Se ha observado que es necesario que la temperatura muestre diferencias cercanas a $10^{\circ} \mathrm{C}$ en el tiempo para 
influir en el ciclo reproductivo y así reflejarse en la condición somática de la almeja, lo cual no se da normalmente en ecosistemas tropicales (Vélez \& Epifanio 1981). Así mismo, la tendencia de la frecuencia de madurez de $P$. solida que varia inversamente con la salinidad, difiere de lo informado para la misma especie en el golfo de Urabá (Duque 1993) y puede ser un reflejo de la correlación inversa del IC y del peso húmedo con la salinidad de esta especie en el SOIS. Esto podría estar obedeciendo a la influencia fluvial tan marcada sobre las lagunas de este estuario, manteniendo el agua en un nivel casi exclusivamente limnético al que la especie se ha adaptado y en el que resulta beneficiada la condición somática de aquellos individuos que habitan en las menores salinidades, como es el caso de las almejas de Poza Verde. Adicionalmente, Cosel (1986) afirma que $P$. solida puede sobrevivir en agua dulce más de tres meses, mientras que en agua típicamente marina, donde no se ha encontrado en el medio natural (Severeyn et al. 1994), solamente sobrevive cuatro semanas.

De lo anterior se puede considerar el hecho de la presencia en Poza Verde de individuos de $P$. solida con un mayor desarrollo de tejido blando en las salinidades más bajas del sistema y donde la influencia del RM es más directa y constante, contrario a lo observado en Atascosa, caracterizada por las salinidades habitualmente más altas y adicionalmente por los aumentos bruscos, como el ocurrido en octubre, que ocasionó una muerte masiva de almejas. Las aperturas de bocas, como la ocurrida en la barra de arena que separa a Atascosa del mar y que produjo este fenómeno, se dan por erosión (Erffa 1973) y ejercen influencia en un área relativamente reducida (Blanco et al. 2006), por eso en las demás ciénagas, más alejadas del límite con el mar, no ocurrieron cambios drásticos de salinidad. Aunque el intercambio con agua de mar normalmente es lento en estos estuarios lagunares, los organismos presentes en una laguna como Atascosa, separada del mar únicamente por una barra de arena relativamente angosta, resultan ser los más sensibles por encontrarse ubicados más cercanos al mar (Olsen et al. 2009).

El crecimiento alométrico negativo en la relación talla-peso total en bivalvos, ha sido asociado con reacciones de defensa ante impactos mecánicos, como la pesca, que conducen a un mayor gasto energético en la producción de concha que de cuerpo (Bald \& Borja 2005). No obstante, la extracción de $P$. solida en el SOIS se lleva a cabo en las tres ciénagas estudiadas; por lo tanto, lo que puede favorecer este tipo de crecimiento, especialmente en Atascosa, podría ser la mayor influencia de las corrientes por la mayor cercanía de esta laguna al mar, haciendo que las almejas desarrollen conchas más gruesas, más pesadas y con una mayor talla promedio, como mecanismo de defensa a la hidrodinámica del ambiente. Esto constituye una situación que puede ir en detrimento de la condición somática de $P$. solida, pero no es el único factor, ya que en El Torno las conchas fueron las más pequeñas y livianas, por lo que es posible que otros factores como la disponibilidad de alimento, estén condicionando pesos relativamente bajos también en el tejido blando (Rueda \& Urban 1998). El Torno presentó la mayor transparencia del agua, lo que puede ser indicativo indirecto de menor concentración de nutrientes (Gocke et al. 2003) y coincide además con el menor promedio de clorofila-a $(8.2 \mu \mathrm{g} / \mathrm{L})$, como se indicó anteriormente.

Por otro lado, el tipo de crecimiento en la relación talla-peso húmedo, se ha asociado al grado de madurez (Le Cren 1951, Gómez 2001). El crecimiento alométrico positivo en Poza Verde, podría ser el reflejo de un mayor porcentaje de individuos maduros en esa ciénaga, mientras que el crecimiento alométrico negativo en Atascosa, podría obedecer a una menor proporción de individuos maduros. Sin embargo esto no explicaría la presencia de crecimiento alométrico positivo en El Torno, cuyo porcentaje de individuos maduros fue aún menor que en Atascosa; las menores tallas y pesos de los individuos en El Torno, pudieron haber influido en este resultado.

Las diferencias entre ciénagas en las variables que describen la condición somática de 
las almejas en este estudio, sumadas a las diferencias en las variables fisicoquímicas de los cuerpos de agua del SOIS, a pesar de estar relativamente cercanos entre sí e interconectados, pueden asociarse a la gran heterogeneidad espacial característica de los estuarios de tipo lagunar, que consisten de un mosaico de hábitats con un gradiente de salinidad marcado de acuerdo con el grado de mezcla del agua dulce con la marina (Olsen et al. 2009). De esta forma, es evidente la importancia de las variables ambientales en la condición de $P$. solida; la salinidad, el $\mathrm{pH}$ y la transparencia del agua, explicaron en conjunto el comportamiento del IC en un porcentaje relativamente alto, resaltando el importante papel del régimen hidrológico en este estuario, con gran afluencia de agua dulce por parte del RM.

Se ha sugerido que los ecosistemas que presentan discontinuidad espacial y diferencias en condiciones ambientales como se evidencia en las tres lagunas del SOIS, exhiben una dinámica parcialmente independiente en aspectos biológicos de los organismos (Reise 2003), como aquellos relacionados con la condición somática de $P$. solida. Debido a los antecedentes de sobreexplotación de la especie (Rueda \& Urban 1998, De La Hoz 2005, 2009), se espera que la presente información tenga utilidad para complementar los resultados de trabajos anteriores en el SOIS y sirva como apoyo para la toma de decisiones por parte de las instituciones encargadas del manejo y conservación del recurso P. solida en el Parque Natural Isla de Salamanca; también se espera que esta información pueda ser aplicable en general a bivalvos de importancia comercial en sistemas lagunares estuarinos del trópico durante los periodos lluviosos.

\section{AGRADECIMIENTOS}

A la Universidad Nacional de Colombia (Posgrado en Biología Marina) y al Instituto de Investigaciones Marinas y Costeras INVEMAR, por su apoyo logístico y financiero. A Efraín Viloria y Carlos Henry por su valiosa colaboración durante el trabajo de campo. A
Néstor Campos, Jacobo Blanco, Efraín Viloria, Héctor Severeyn, Cristian Ituarte y Mercedes Durfort, por su asesoría y sus sugerencias en el análisis de la información del documento.

\section{RESUMEN}

Se evaluó la condición somática de la almeja Polymesoda solida en tres lagunas del Parque Isla de Salamanca (abril a noviembre de 2004), durante el periodo lluvioso. En 60 individuos por laguna, se analizaron mensualmente índices de condición, rendimiento, relación talla-peso y madurez gonadal en fresco. También se midió salinidad, temperatura, $\mathrm{pH}$, oxígeno disuelto, transparencia y profundidad del agua. Los mayores índices de condición coincidieron con descensos en el nivel del río Magdalena, especialmente en Poza Verde y El Torno. Estos índices fueron más altos en tallas entre $27 \mathrm{~mm}$ y $33 \mathrm{~mm}$, donde también se ubicó la talla media de madurez sexual. En Poza Verde, laguna con mayor influencia del río, se presentaron los mayores índices de condición, rendimiento, peso húmedo y frecuencia de madurez, revelando una mejor condición de los organismos. El índice de condición (peso cuerpo/ peso concha) presentó mayor coeficiente de regresión múltiple con la salinidad, $\mathrm{pH}$ y transparencia, sugiriendo la importancia del régimen hidrológico en su regulación. Las diferencias espaciales en las variables evaluadas, resaltan la necesidad de tener en cuenta la heterogeneidad de este tipo de estuarios para la conservación y manejo adecuado de la pesquería de $P$. solida.

Palabras clave: Polymesoda solida, bivalvos, Isla de Salamanca, estuarios, régimen hidrológico, índice de condición.

\section{REFERENCIAS}

Acosta, V., A. Prieto \& C. Lodeiros. 2006. Índice de condición de los mejillones Perna perna y Perna viridis (Bivalvia: Mytilidae), bajo un sistema suspendido de cultivo en la Ensenada de Turpialito, Golfo de Cariaco, Venezuela. Zootecnia Trop. 24: 177-192.

Báez, M., Y. García \& H. J. Severeyn. 2005. Ciclo reproductivo de Geukensia demissa en la playa de Nazaret, El Moján, Estado Zulia, Venezuela. Cienc. Mar. 31: 111-118.

Bald, J. \& A. Borja. 2005. Estudio del estado de los recursos de almeja y berberecho en los estuarios Mundaka, Plentzia y Txingudi. Informe Técnico No. 105. Departamento de Agricultura y pesca. Servicio Central de Publicaciones del Gobierno Vasco, VitoriaGasteiz, País Vasco, España (también disponible en línea: www.azti.es/muestracontenido.asp?idcotenido $=272 \&$ content $=8 \&$ nodo $1=26 \&$ nodo $2=0$ ). 
Bautista, C. 1989. Moluscos. Tecnología de cultivo. Mundiprensa, Madrid, España.

Bernal, G. 1996. Caracterización geomorfológica de la Llanura Deltaica del Río Magdalena, con énfasis en el sistema lagunar de la Ciénaga Grande de Santa Marta, Colombia. Bol. Invest. Mar. Cost. 25: 19-48.

Blanco, J.A., E.A. Viloria \& J.C. Narváez. 2006. ENSO and salinity changes in the Ciénaga Grande de Santa Marta coastal lagoon system, Colombian Caribbean. Est. Coast. Shelf. Sci. 66: 157-167.

Botero, L., J.E. Mancera-Pineda, L.A. Vidal, A. SantosMartínez, G. Ramírez, M.L. Fontalvo, L.F. Espinosa, W. Troncoso, E. Viloria \& J.G. Salazar. 1995. Informe sobre la mortandad masiva de peces ocurrida en el complejo lagunar Ciénaga Grande de Santa Marta-Caribe colombiano, en junio de 1995. Progr. Lagunas Costeras, INVEMAR, Santa Marta, Magdalena, Colombia.

Bulger, A. J., B. P. Hayden, M. E. Monaco, D. M. Nelson \& M. G. McCormick. 1993. Biologically-based estuarine salinity zones derived from a multivariate analysis. Estuaries 16: 311-322.

Cabrera, J. H., M. Protti, M. Urriola \& O. Sáenz. 2001. Crecimiento y madurez sexual de una población de Saccostrea palmula (Mollusca: Bivalvia), Costa Rica. Rev. Biol. Trop. 49: 877-882.

CORPAMAG-UNINORTE. 2005. Alternativas agroambientales de la zona Sur de la Ecorregión Ciénaga Grande de Santa Marta. Santa Marta, Magdalena, Colombia.

Cosel, R. Von. 1986. Moluscos de la región de la Ciénaga Grande de Santa Marta (costa del Caribe de Colombia). An. Inst. Inv. Mar. Punta Betín 15-16: 79-370.

De La Hoz, M. V. 2005. Distribución, abundancia y aspectos biológicos de la almeja Polymesoda solida Phillippi, 1846 (Bivalvia: Corbiculidae) en la Isla de Salamanca, Caribe colombiano. Tesis MSc., Universidad Nacional de Colombia, Santa Marta, Magdalena, Colombia.

De La Hoz, M. V. 2009. Densidad, estructura de tallas y explotación pesquera del bivalvo Polymesoda solida en un sistema lagunar del Caribe colombiano. Bol. Centro Invest. Biol. 43: 1-27.

Díaz, J.M. \& M. Puyana. 1994. Moluscos del Caribe colombiano. Un catálogo ilustrado. COLCIENCIAS, Fundación Natura, INVEMAR, Bogotá D. C., Colombia.

Duque, P. 1993. Algunos aspectos de la biología y ecología de Polymesoda arctata (almeja) en la Bahía de Mari- rrio (Golfo de Urabá). Tesis Biología, Universidad de Antioquia, Medellín, Antioquia, Colombia.

Erffa, A. Von. 1973. Sedimentación, transporte y erosión en la costa Norte de Colombia entre Barranquilla y la Sierra Nevada de Santa Marta. Mitt. Inst. ColomboAlemán Invest. Cient. 7: 155-209.

García, C. 1997. Biología, ecología y aspectos de cultivo del hacha Pinna carnea (Gmelin, 1791) (Mollusca, Bivalvia, Pinnidae) en la región de Santa Marta, Caribe colombiano. Tesis Biología Marina, Universidad Jorge Tadeo Lozano, Santa Marta, Magdalena, Colombia.

García, Y., H. J. Severeyn \& J. J. Ewald. 1994. Early development of the estuarine mollusk Polymesoda solida (Philippi, 1846) (Bivalvia: Corbiculidae) in Lake Maracaibo. Venezuela. Am. Malacol. Bull. 11: 51-56.

Gocke, K., J.E. Mancera, L. A. Vidal \& D. Fonseca. 2003. Planktonic primary production and community respiration in several coastal lagoons of the outer Delta of the Río Magdalena, Colombia. Bol. Invest. Mar. Cost. 32: 93-123.

Gómez, P. 2001. Identificación de stocks de pargo rayado Lutjanus synagris (Linnaeus, 1758) en el área norte del Caribe colombiano. Tesis Biología Marina, Universidad Jorge Tadeo Lozano, Santa Marta, Magdalena, Colombia.

Hernández, C.A. 1983. Estado actual de los bancos naturales de Crassostrea rhizophorae Guilding 1828 en el norte de la Ciénaga Grande de Santa Marta. Tesis Biología, Universidad Nacional de Colombia, Bogotá D.C., Colombia.

Hernández, C.A. 1988. Características físicas, químicas y biológicas de la Ciénaga Grande de Santa Marta, Colombia (2a parte). Instituto de Investigaciones Marinas y Costeras (INVEMAR), Santa Marta, Magdalena, Colombia.

Holme N.A. \& A.D. McIntyre. 1971. Methods for the study of marine benthos. Blackwell Scientific Oxford, Inglaterra.

IDEAM. 2003. Promedios mensuales de caudal del Río Magdalena. Instituto de Hidrología, Meteorología y Estudios Ambientales IDEAM. Estación de Calamar, Atlántico, Colombia.

INVEMAR. 2002. Informe del estado de los ambientes marinos y costeros de Colombia. Serie de Publicaciones Especiales No. 8. Instituto de Investigaciones Marinas y Costeras (INVEMAR), Santa Marta, Magdalena, Colombia. 
INVEMAR. 2004. Monitoreo de las condiciones ambientales y los cambios estructurales y funcionales de las comunidades vegetales y de los recursos pesqueros durante la rehabilitación de la Ciénaga Grande de Santa Marta. Instituto de Investigaciones Marinas y Costeras (INVEMAR), Santa Marta, Magdalena, Colombia.

INVEMAR. 2008. Monitoreo de las condiciones ambientales y los cambios estructurales y funcionales de las comunidades vegetales y de los recursos pesqueros durante la rehabilitación de la Ciénaga Grande de Santa Marta. Informe técnico final 2008. Instituto de Investigaciones Marinas y Costeras. Santa Marta, Magdalena, Colombia.

INVEMAR-CORPAMAG. 2002. Monitoreo de las condiciones ambientales del Sector Occidental de la Isla de Salamanca. Descripción de la calidad ambiental. Instituto de Investigaciones Marinas y Costeras (INVEMAR), Santa Marta, Magdalena, Colombia.

King, M. 1995. Fisheries biology, assessment and management. Fishing News Books, Oxford, Inglaterra.

Le Cren, E. 1951. The length-weight relationship and seasonal cycle in gonad weight and condition in the pearch (Perca fluviatilis) J. Anim. Ecol. 20: 201-219.

Olsen, S. B., T. V. Padma \& B. D. Ritcher. 2009. Guía para el manejo del agua dulce a los estuarios. Agencia de los Estados Unidos para el desarrollo internacional, Washington D. C., EEUU (también disponible en línea: www.nature.org/initiatives/freshwater/files/ tnc_methodsguide_span_final.pdf).

Ospina, D. 2001. Introducción al muestreo. Unibiblos, Universidad Nacional de Colombia, Bogotá D. C., Colombia.

Prieto, A., O. A. Ramos, D. Arrieche, J. Villalba \& C. Lodeiros. 2001. Producción secundaria e índice de condición en Arca zebra (Mollusca: Bivalvia) del Golfo de Cariaco, Venezuela. Rev. Biol. Trop. 49: 599-608.

Reise, K. 2003. Metapopulation structure in the lagoon cockle Cerastoderma lamarcki in the Northern Wadden Sea. Helgol. Mar. Res. 56: 252-258.

Rueda, M. \& H.J. Urban. 1998. Population dynamics and fishery of the freshwater clam Polymesoda solida (Corbiculidae) in Cienaga Poza Verde, Salamanca Island, Colombian Caribbean. Fish. Res. 39: 75-86.

Ruiz, E., E. Cabrera, P.R. Cruz \& J.A. Palacios. 1998. Crecimiento y ciclo reproductivo de Polymesoda radiata en Costa Rica. Rev. Biol. Trop. 46: 643-649.
Santos-Martínez, A. \& N. Bateman. 1997. Evaluación de los principales recursos pesqueros de la Ciénaga Grande de Santa Marta. Informe programa Calidad Ambiental Marina. Proyecto DELTA, INVEMAR, Santa Marta, Magdalena, Colombia.

Schweers, T., M. Wolff, V. Koch \& F. S. Duarte. 2006. Population dynamics of Megapitaria squalida (Bivalvia: Veneridae) at Magdalena Bay, Baja California Sur, Mexico. Rev. Biol. Trop. 54: 1003-1017.

Severeyn, H.J., Y. García \& J.J. Ewald. 1994. Taxonomic revision of Polymesoda solida (Phillippi, 1846) (Bivalvia: Corbiculidae), a new name for Polymesoda arctata, the estuarine clam of Lake Maracaibo and other estuaries of the tropical Atlantic coasts of America. Ciencia 2: 53-65.

Silva, A.M. \& R. Bonilla. 2001. Abundancia y morfometría de Anadara tuberculosa y A. similis (Mollusca: Bivalvia) en el manglar de Purruja, Golfo Dulce, Costa Rica. Rev. Biol. Trop. 49: 315-320.

Sokal, R. \& F. J. Rohlf. 1981. Biometry. W.H. Freeman, Nueva York, EEUU.

Solano, Y., J. Cabrera, J.A. Palacios \& R. A. Cruz. 1997. Madurez sexual, índice de condición y rendimiento de Pinctada mazatlanica (Pterioida: Pteriidae), Golfo de Nicoya, Costa Rica. Rev. Biol. Trop. 45: 10491054.

Sparre, P. \& S.C. Venema. 1995. Introducción a la evaluación de recursos pesqueros tropicales. Parte 1. Manual FAO, Valparaíso, Chile.

Squires, M., M. Estévez, O. Barona \& O. Mora. 1978. Mangrove cockles, Anadara sp. (Mollusca: Bivalvia) of the Pacific coast of Colombia. The Veliger 18: 57-68.

Vélez, A. \& C.E. Epifanio. 1981. Effects of temperature and radiation on gametogenesis and growth in the tropical mussel Perna perna (L.). Aquaculture 22: 21-26.

Zar, J. H. 1999. Biostatistical analysis. Prentice Hall, Nueva Jersey, EEUU.

\section{REFERENCIAS DE INTERNET}

SIBM. 2009. Sistema de Información sobre Biodiversidad Marina de Colombia. Instituto de Investigaciones Marinas y Costeras INVEMAR. Santa Marta, Magdalena, Colombia. (Consultado abril 2009, www. invemar.og.co/siam/sibm/index.htm). 
